\title{
Photon penetration depth in human brain for light stimulation and treatment: A realistic Monte Carlo simulation study
}

\author{
Ting $\mathrm{Li}^{*} \S$, Chang Xue ${ }^{\dagger}$, Pengbo Wang ${ }^{\dagger}$, Yan $\mathrm{Li}^{\star}$ and Lanhui $\mathrm{Wu}^{\dagger}$ \\ *Institute of Biomedical Engineering \\ Chinese Academy of Medical Science and \\ Peking Union Medical College \\ 300192, Tianjin, P. R. China \\ ${ }^{\dagger}$ State Key Lab Elect Thin Film \& Integrated Device \\ and Department of Biomedical Engineering \\ University of Electronic Science \& Technology of China \\ Chengdu 610054, P. R. China \\ *Design Center \\ Avic Beijing Keeven Aviation Instrument Co., Ltd \\ China Aviation Industry Corporation \\ Beijing 100098, P. R. China \\ \$litinghaha9@126.com
}

Received 24 February 2017

Accepted 18 May 2017

Published 11 July 2017

\begin{abstract}
Light has been clinically utilized as a stimulation in medical treatment, such as Low-level laser therapy and photodynamic therapy, which has been more and more widely accepted in public. The penetration depth of the treatment light is important for precision treatment and safety control. The issue of light penetration has been highlighted in biomedical optics field for decades. However, quantitative research is sparse and even there are conflicts of view on the capability of near-infrared light penetration into brain tissue. This study attempts to quantitatively revisit this issue by innovative high-realistic 3D Monte Carlo modeling of stimulated light penetration within high-precision Visible Chinese human head. The properties of light, such as its wavelength, illumination profile and size are concern in this study. We made straightforward and quantitative comparisons among the effects by the light properties (i.e., wavelengths: 660,810 and $980 \mathrm{~nm}$; beam types: Gaussian and flat beam; beam diameters: 0, 2, 4 and $6 \mathrm{~cm}$ ) which are in the range of light treatment. The findings include about $3 \%$ of light dosage within brain tissue; the combination of Gaussian beam and $810 \mathrm{~nm}$ light make the maximum light penetration
\end{abstract}

$\S_{\text {Corresponding author. }}$

This is an Open Access article published by World Scientific Publishing Company. It is distributed under the terms of the Creative Commons Attribution 4.0 (CC-BY) License. Further distribution of this work is permitted, provided the original work is properly cited. 
$(>5 \mathrm{~cm})$, which allows light to cross through gray matter into white mater. This study offered us, the first time as we know, quantitative guide for light stimulation parameter optimization in medical treatment.

Keywords: Penetration depth; light therapy; light propagation; MCVM; human brain.

\section{Introduction}

The medical photonics research has come to the foreground in recent years. Light stimulation has been applied into medical treatment study, such as low-level laser therapy and photodynamic therapy, have experienced a rapid development from experimental stage to practical stage. ${ }^{1,2}$ While the temperature variation or heat generation effect has been well studied in the research of light therapy, the quantitative knowledge of light propagation and penetration depth is rare. ${ }^{3}$ There is no doubt that for those light stimulation and treatment fields, the penetration depth of light is critical for both research and clinical application, especially on the issue for precision of personal medicine, quantitative-guide treatment, optimization of the light therapy parameters, and safety control. There is sparse experiment study on light penetration depth for human structured tissues, only semi-infinite phantom or layered phantom study were published. ${ }^{4}$

However, it is undoable to measure, by experiment, light penetration depth within human head or mimicking head model with the complex geometry and structure. The alternative way is precise modeling of light propagation within realistic human head model. The diffuse equation method and Monte Carlo modeling are the main ways to obtain the computation of light propagation. Compared to the recent developed analytical approximation method, ${ }^{5}$ Monte Carlo method is more flexible and reliable, especially for noninfinite medium. There are several widely used Monte Carlo simulation techniques offered in our field, such as the one for layered tissue model (MCML) ${ }^{6}$ the one for medical image depicted tissue model (tMCimg) ${ }^{7}$ the one for mathematical geometry depicted tissue model (MCLS), ${ }^{8}$ and the one for $3 \mathrm{D}$ voxelized media (MCVM). ${ }^{9}$ Although these methods have been accepted in biomedical field in accurate light propagation computation, the MCML and MCLS are only applicable layered tissue model or the model depicted with cube, sphere, or cylinder. Only tMCimg and MCVM are capable of modeling light propagation within realistic structure tissue model. MCVM is most fully tested. ${ }^{6}$

Brain is of great significance in biomedical optics field, not only because of its role in functional activities and intelligence study, but also because of its numerous complex disease requiring searching for novel therapy and the complexity of those therapy methods. ${ }^{10-12}$ Especially, in the field of light stimulation and therapy, the most meaningful, challenging, and mostly-researched part of human body is human brain. ${ }^{12-14}$ While the other parts of human body, like breast, leg, arm, and belly, are simple in structure which mostly can be treated as layered model, the head is of complicate three-dimensional (3D) structure and complex geometry of cerebral tissue. It is not convincing to use layered head model to study light penetration depth. Realistic human brain structure model is required to provide reliable enough estimation of light penetration depth.

Recently, there was some realistic Monte Carlo simulation study which analyzed the factors affecting light propagation in realistic human brain, however most of them were not focused on the penetration depth and failed in offering quantitative comparison or answers, not to mention the effect of treatment light properties on light penetration depth. ${ }^{8,10,11,15}$ There was only one Monte Carlo study on light penetration depth on realistic head model, ${ }^{13}$ which uses a MRI head model in which precision is not high enough. A more realistic and precised head model was recommended to model light propagation. ${ }^{11}$

Visible human projects of the world provided us the realistic structured human brain model. ${ }^{16}$ Thereinto, Visible Chinese human (VCH) is famous in highest-resolution structure image dataset, finest and professional segmentation of $\mathrm{VCH}$ into different types of tissues. ${ }^{16,17} \mathrm{VCH}$ head dataset is capable to provide us the highest realistic human model for us to study particle propagation, ${ }^{11,15,18}$ which delight us to study light penetration depth with $\mathrm{VCH}$ head model. Of note, the $\mathrm{VCH}$ model is a $3 \mathrm{D}$ voxelized 
model, requiring us to simulate light transportation within human head with a Monte Carlo approach which is compatible to voxelized media.

Our study aims to obtain quantitative evaluation of light penetration depth for light stimulation and therapy. We managed to use the most-realistic human head model, $\mathrm{VCH}$, and the most reliable 3D Monte Carlo modeling technique which is compatible for the VCH voxelized model, MCVM to modeling 3D light propagation in human head. The important illumination parameters, such as the wavelength, profile, and beam size of the treatment light, are interrogated to study the quantitative effect of those parameters to light penetration depth. The data of those parameters are set in the range of commonly used. ${ }^{15}$ The percentage of light fluence in different-depth tissues, the contoured pseudo color map of fluence distribution, the sampled fluence decline with penetration depth, and the accumulated number of light-penetrated voxels are used to scale the light penetration depth.

This study finally provided numeric evaluation of light penetration depth and quantitative comparison of the commonly-used treated light properties on light penetration. The findings showed deep penetration of light within gray matter and white matter, which support the capabilities of light stimulation and treatment in cerebral tissue volume. Plus the quantitative comparison on the illumination parameters based on our MCVM. VCH modeling exampled a reliable, fast, low-cost, and no-animal-test way to obtain light penetration depth value estimates for light stimulation and light therapy, compared to previous simplistic phantom study, simulation studies, animal therapeutic effect measurement studies.

\section{Methods and Materials}

\subsection{VCH brain model}

The VCH head model we used contains various types of tissues in a Chinese male human whole body frozen in the standing posture, and was horizontally sectioned at $0.02 \mathrm{~cm}$ interval. $0.01 \mathrm{~cm}$-perpixel-resolution photographs were obtained with digital color, which is higher than $\mathrm{CT}$ and MRI. The sections of $\mathrm{VCH}$ are performed by color photographs (see Fig. 1(a)). Distinct tissues in each slice were visibly distinguished, segmented and labeled with a series of specified number (see Fig. 1(b)). These precisely segmented and labeled sections and high-resolution colored photographs make it more realistic for studying human brain. Besides, compared to CT and MRI, which require lying posture, the upstanding posture in $\mathrm{VCH}$ is more close to the natural and real geometry of brain surface for the research about light penetration prediction. ${ }^{10}$

In the analysis of the color photographs and the numbered sections, the head model was segmented into eight types of tissue, including scalp, skull, CSF, muscle, visible artery, vein blood vessels, gray and white matter. We chose every four continuous pixels in both anterior-posterior and left-right directions in two continuous slices to form voxels of $0.04 \times 0.04 \times 0.04 \mathrm{~cm}^{3}$. This was the voxel size used to balance the computation parts in the simulation and get it more precise and real. Finally, we got a $450 \times 400 \times 150$ voxel-size three-dimension matrix which contains a numerical scale, a list of various tissue types. The list describes the optical features of each specific tissue for each voxel. The optical features of these tissues at 660, 810 and $980 \mathrm{~nm}^{10,15,19,20}$ are listed in Table 1.

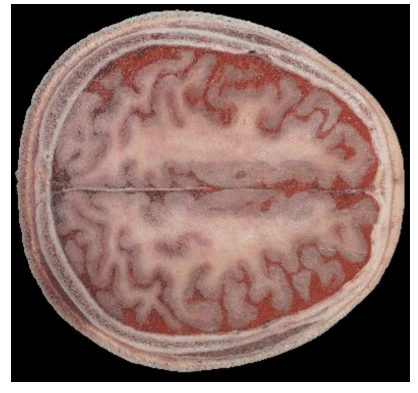

(a)

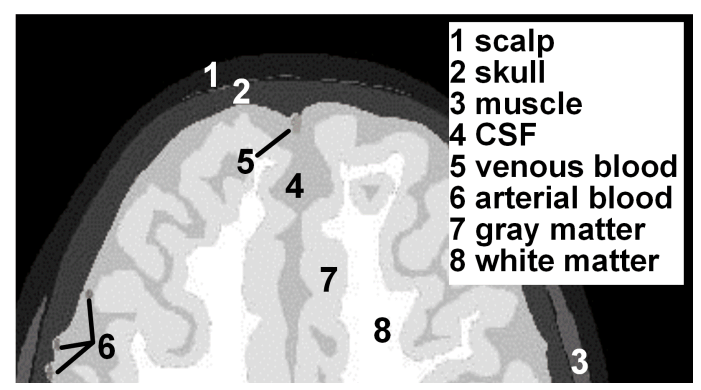

(b)

Fig. 1. VCH head model. (a) Digital color photograph of one typical slice in the VCH head dataset; (b) Segmentation of the slice in (a) and identification of different types of tissues. 
Table 1. Optical properties of head tissues for 660,810 and $980 \mathrm{~nm}$ light. The units of both absorption coefficient $\left(\mathrm{u}_{\mathrm{a}}\right)$ and scattering coefficient $\left(\mathrm{u}_{\mathrm{s}}\right)$ are $\mathrm{cm}^{-1}$.

\begin{tabular}{lccc}
\hline $\begin{array}{l}\text { Wavelength } \\
(\mathrm{nm})\end{array}$ & $\begin{array}{c}660 \\
\mathrm{u}_{\mathrm{a}} / \mathrm{u}_{\mathrm{s}}\end{array}$ & $\begin{array}{c}810 \\
\mathrm{u}_{\mathrm{a}} / \mathrm{u}_{\mathrm{s}}\end{array}$ & $\begin{array}{c}980 \\
\mathrm{u}_{\mathrm{a}} / \mathrm{u}_{\mathrm{s}}\end{array}$ \\
\hline Scalp & $0.340 / 25.8$ & $0.195 / 19.2$ & $0.450 / 14.0$ \\
$\mathrm{CSF}$ & $0.040 / 3.5$ & $0.050 / 2.4$ & $4.5 / 1$ \\
Skull & $0.300 / 24.3$ & $0.165 / 15.6$ & $0.360 / 11.5$ \\
Muscle & $0.868 / 8.61$ & $0.273 / 6.87$ & $0.547 / 5.65$ \\
Gray matter & $0.22 /(88 \times(1-0.85))$ & $0.3 /(70 \times(1-0.892))$ & $0.5 /(55 \times(1-0.9))$ \\
White matter & $0.82 /(400 \times(1-0.835))$ & $1 /(350 \times(1-0.862))$ & $1.4 /(300 \times(1-0.88))$ \\
Arterial blood & $1.30 / 61.1$ & $2.33 / 50$ & $2.90 / 37.4$ \\
Venous blood & $4.87 / 25.0$ & $2.38 / 52.2$ & $1.62 / 5.15$ \\
\hline
\end{tabular}

\section{2. $M C V M$}

The software we used was MCVM programmed by Ting $\mathrm{Li}$, which applied to $3 \mathrm{D}$ voxelized media. The detailed algorithm has been described in Ref. 6 . Briefly, photon propagation in the head was determined by several factor such as scattering coefficient, absorption coefficient, phase function, reflections and refractions in the 3D arbitrary surface of the tissue and complex interfaces between different types of tissue. This program checked whether a photon could travel through a boundary between different tissues once encountered a substep. Reflection and refraction were also considered in this program. Each actual substep was relative to a single voxel and a single absorption coefficient, and thus the absorption related with such substep was recorded precisely to the local voxel by pseudo continuous absorption weighting. So it is with scattering coefficients. It proves that MCVM has been validated well with both theoretical data and experimental data.

In the simulation, we set up the input and output file name, the illumination position and directions, number of photons as well as the optical properties of each type of tissue included. After the runs of MCVM simulation, the outputted 3D light fluence distribution were extracted out for analysis.

\subsection{Simulation}

The input data based on Table 1, was set in MCVM simulations. After computation, the results about the light absorption within different voxels were output and extracted out. After simulations, we calculated the fluence distribution, divided the output light absorption distribution by the absorption coefficient distribution in the tissue. With statistical analysis on the characteristic parameters on the 3D fluence distribution, we obtained the evaluation of light penetration depth at different values of beam types, wavelengths and sizes. The position in the center of forehead, which is $1 \mathrm{~cm}$ above the eyebrow, is ideal to place the center of beam and the inject direction of it is just the normal direction of the forehead. The comparison within those results were performed to address the effect of wavelengths, beam types and beam sizes.

The wavelength, beam type and beam size are the main factors affecting light fluence distribution in human brain in consideration of this study. We chose three wavelengths: 660,810 and $980 \mathrm{~nm}$ with the same beam size, two beam types: Gaussian beam and Top-hat beam and three kinds of beam size different in diameters: 2,4 and $6 \mathrm{~cm}$ with the same wavelength of $810 \mathrm{~nm}$, totally 12 simulations. The number of photons in each simulation are $10^{7}$ in total, which means the beams in our simulation study have same power density (normalized to be $1 \mathrm{~W})$. Each simulation was repeated for 10 times and the result was obtained in average for data analysis to increase the signal-to-noise ratio.

\subsection{Data analysis}

The results of 10 times simulation of the 3D fluence distribution at different combinations of treated light parameters were averaged by 10 to eliminate random effect. Then, the percentage of light fluence in different-depth tissues were analyzed with pie chart. The contoured pseudo color map of fluence distribution were displayed to analyze the penetration depth visibly in space. In those photographs, different colors, such as red, orange, yellow, blue and purple, represent the output light absorption 
distribution according to their intensity. The red means the highest absorption distribution, the orange means second high and so on. The sampled fluence decline at the light source emitting direction were extracted to show its decrease with penetration depth. The number of light-penetrated voxels are accumulated to investigate the penetrated depth scale especially in gray and white matter.

The slice numbered 269 was exactly where the beam source center was placed, which is $1 \mathrm{~cm}$ above the eyebrow. Therefore, when analyzing the final results, we chose the color photographs of this slice in presentation. To make our research more accurate and intuitive, we accumulated all the fluence in brain and then calculated the proportion of each tissue in the form of percentage, and made a pie chart about the fluence distribution in brain tissues according to those research.

\section{Results}

\subsection{Light fluence distribution}

The pie chart (Fig. 2) suggests the percentages of fluence in each tissue that we detected in brain, such as scalp, scull, CSF, etc., which were close to the simulation results shown in Ref. 15. It supported the reliability of the simulation. Moreover, the percentage of fluence in gray and white matter proved the light penetration is deep into cerebral tissue.

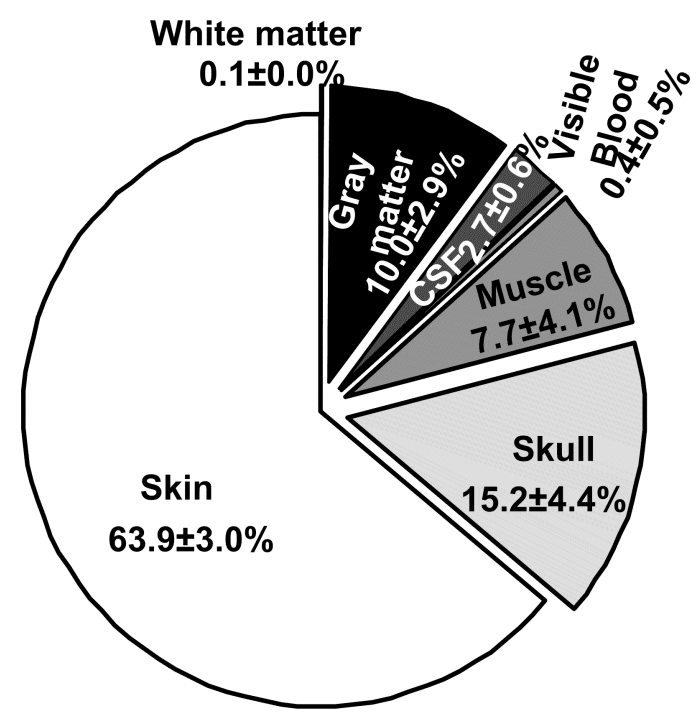

Fig. 2. Percentage of light fluence distribution in each type of tissue in VCH head model under normal condition. Data are shown in average statistics among different emitting positions on the forehead.
Figure 3 intuitively showed the different abilities of each type of light in penetration into brain. From the results, we can get some idea of the approximate area of the light penetration inside human brain, which includes white matter and gray matter. In Fig. 3(a), it is obvious that wavelength plays a more important role in determining light penetration depth than beam type. It is obviously found that $810 \mathrm{~nm}$ is more suitable than $660 \mathrm{~nm}$ and $980 \mathrm{~nm}$ in transcranial LLLT for more deeply and widely penetration. Clearly, for $810 \mathrm{~nm}$, red colored fluence has reached the gray matter and yellow colored fluence has even well reached the interior white matter. On the contrary, for $660 \mathrm{~nm}$, yellow colored fluence only reached the gray matter and indigo colored fluence reached the white matter. While for $980 \mathrm{~nm}$, only blue and purple parts could reach the white matter. Besides, It is found that for $810 \mathrm{~nm}$, light could reach more widespread region in human brain compared to the other two wavelengths. For $980 \mathrm{~nm}$, penetrated light is most concentrated but could not reach very deeply in brain, and thus it is ideal for light acupuncture, which requires highly focused and precisely located penetrating beam. For phototherapy, if doctors need to stimulate deeply into cerebral tissue, they would better choose $810 \mathrm{~nm}$. Compared to the other two, $660 \mathrm{~nm}$ is basically a modest choice.

Another important finding is that beam type plays a less important role in affecting the penetration depth of light in human brain. There is slight difference between Gaussian and Top-hat beams in affecting light fluence distribution. It is showed that Gaussian beams was able to penetrate a little more deeply than Top-hat beams, but the difference is very slight. For example, for $810 \mathrm{~nm}$, it could be distinguished that for the photograph of Top-hat beam, yellow part took more area of white matter compared to Gaussian beam.

The result of simulations based on two beam types (Gaussian and Top-hat) and three diameters $(2,4$ and $6 \mathrm{~cm})$ is shown in Fig. 3(b). There is a tendency that as the beam size decreases, beam could penetrate more widely and deeply inside human brain. On the other hand, it could be found that when beam size is the same, beam type hardly causes difference between these two simulations. Accordingly, compared to beam type, beam size has more influence on the penetration depth of light. Another interesting phenomenon in those photographs is that as the beam size increases, the area 


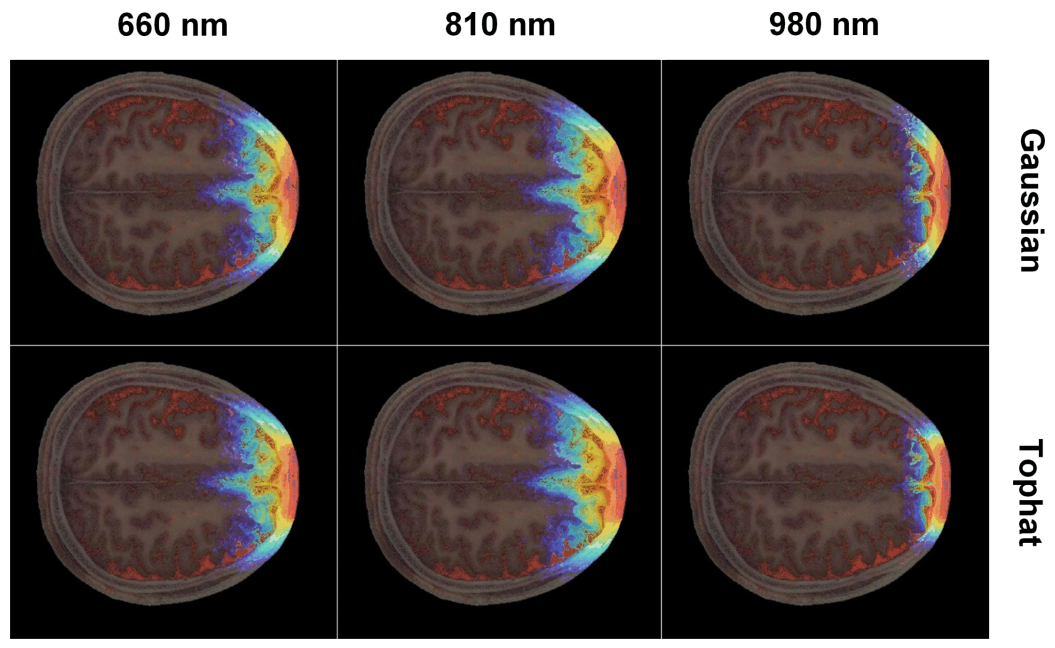

(a)

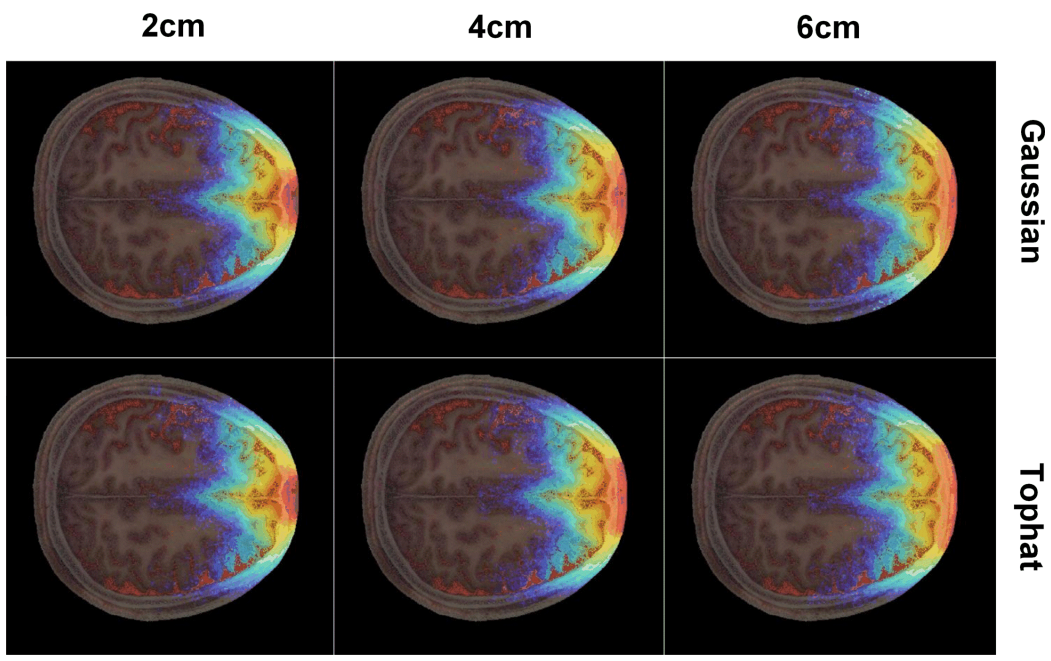

(b)

Fig. 3. Light fluence distribution with the background of head geometry and 3D structure. The result in the slice numbered 269 are shown. (a) The LLLT fluence distribution respective to Gaussian and Top-hat beams at different wavelengths (660, 810 and $980 \mathrm{~nm})$; (b) The LLLT fluence distribution respective to Gaussian and Top-hat beams of different diameters (2, 4 and $6 \mathrm{~cm})$.

beams could reach increase slightly, but the difference is not significant and the quantitative analysis were offered below.

\subsection{Penetration depth}

Figures 4 and 5 provide researchers sampled fluence decline with penetration depth and the exact penetration depth of several commonly used beams, and they also provide comparison among those beams. The horizontal dotted line, corresponding to, represents the penetration depth most equipment available now that could measure accurately. And the horizontal dotted line, corresponding to, represents the limiting penetration depth which the most advanced equipment could measure so far.

In Fig. 4, for beams of the same wavelength, Gaussian beams was able to penetrate more deeply than Top-hat beams, although the difference is relatively small, which is coincident with the above figures. For example (as shown in Tables 2 and 3), the penetration depth of Top-hat beams is approximately $3.81 \mathrm{~cm}$ while the penetration depth of Gaussian beams is approximately $4.13 \mathrm{~cm}$ at the dotting line scales. The differences for $980 \mathrm{~nm}$ (although it is not very obvious on this chart) and $660 \mathrm{~nm}$ are the same in this photograph. Besides, for beams of the same beam type, the penetration 


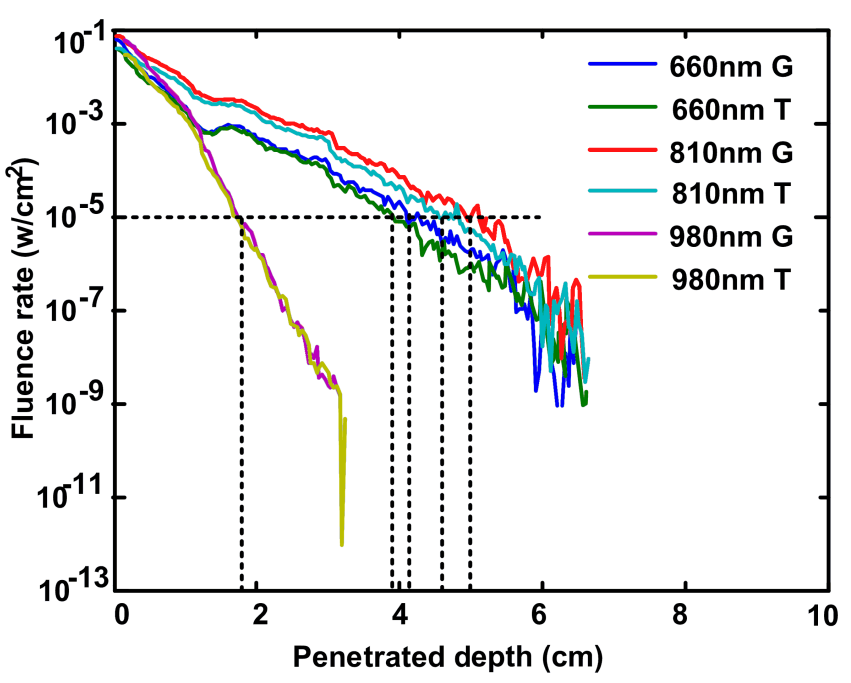

Fig. 4. The fluence decline following with the penetrated depth from the beam center into head tissue at normal light incidence direction. $\mathrm{G}$ and $\mathrm{T}$ denote to Gaussian and Top-hat beams respectively. Wavelengths 660,810 and $980 \mathrm{~nm}$ are included.

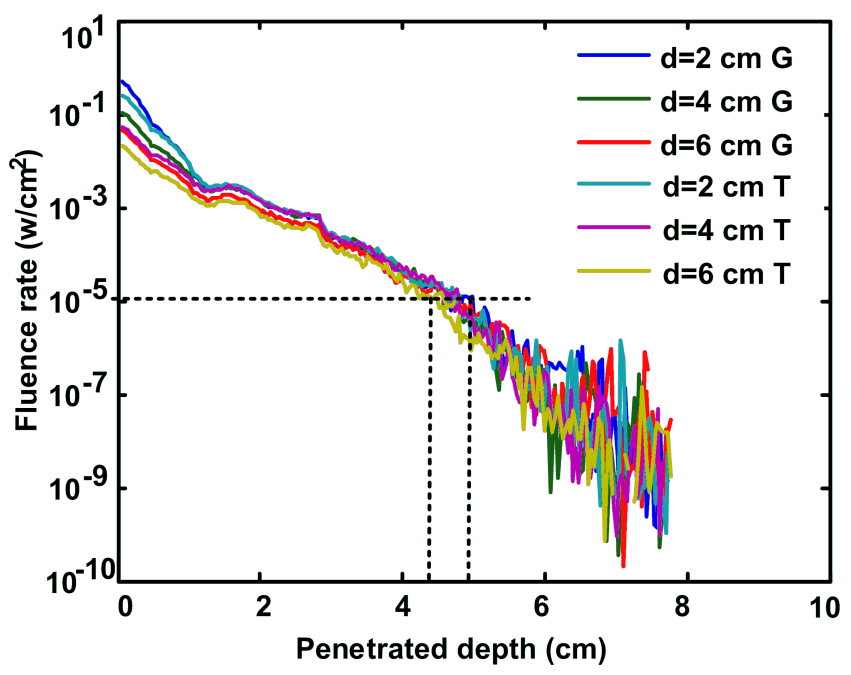

Fig. 5. The fluence decline following with the penetrated depth from the beam center into head tissue at normal light incidence direction. $\mathrm{G}$ and $\mathrm{T}$ denote to Gaussian and Top-hat beams, respectively. depth of $810 \mathrm{~nm}$ is the greatest, which reaches $4.13 \mathrm{~cm} .660 \mathrm{~nm}$ takes the second in rank, reaching $3.45 \mathrm{~cm}$ in depth. $980 \mathrm{~nm}$ obtained the smallest penetration depth, which is only about $1.45 \mathrm{~cm}$.

In Fig. 5, the penetrated fluence attenuation of those six types of beams in light incidence direction were extracted precisely. If carefully observing the positions of those intersection points of those colored lines and the dotted line, it could be found that for both beam type, the penetration depth of light decreases as the beam size increases. Besides, it is hard to distinguish the difference between the penetration depths of beams with the same beam size but different beam types.

\subsection{Number of light penetrated voxels in cerebral tissues}

The number of penetrated voxels by light is an overall parameter to reflect the capability of beam penetration in human brain. As shown in Fig. 6, white bars represented the number of voxels in the gray matter reached by light and black bars represented the number of voxels in the white matter reached by light. It is obvious that for $810 \mathrm{~nm}$, the numbers of light penetrated voxels within cerebral tissues were much larger than the other two wavelengths. This simulation results could also verify that $810 \mathrm{~nm}$-light could penetrate most deeply and widely within cerebral tissue. Additionally, for both $810 \mathrm{~nm}$ and $660 \mathrm{~nm}$, Top-hat beam showed a slightly $(1-2 \%)$ better performance in penetrating inside brain, but for $980 \mathrm{~nm}$, the opposite. Besides, the different effects beam type brings are obviously very slight, which is the same with our previous findings.

Different from Figs. 6(a) and 6(b) showed that Top-hat beam always performed better in penetrating within cerebral tissue, compared to Gaussian beam. Plus, as beam diameter increases, the number of penetrated voxels increases as well, and

Table 2. The penetration depth value extracted from Fig. 5. G means Gaussian beam; T means Top-hat beam.

\begin{tabular}{|c|c|c|c|c|c|c|c|}
\hline \multirow{2}{*}{\multicolumn{2}{|c|}{ Wavelength (nm) }} & \multicolumn{2}{|c|}{660} & \multicolumn{2}{|c|}{810} & \multicolumn{2}{|c|}{980} \\
\hline & & $\mathrm{G}$ & $\mathrm{T}$ & $\mathrm{G}$ & $\mathrm{T}$ & $\mathrm{G}$ & $\mathrm{T}$ \\
\hline \multirow[t]{2}{*}{ Penetration depth $(\mathrm{cm})$} & Fluence $=10^{-5} \mathrm{w} / \mathrm{cm}^{2}$ & 3.45 & 3.28 & 4.13 & 3.81 & 1.45 & 1.4 \\
\hline & Fluence $=10^{-7} \mathrm{w} / \mathrm{cm}^{2}$ & 4.71 & 4.93 & 5.13 & 5.01 & 1.95 & 2.04 \\
\hline
\end{tabular}


Table 3. The penetration depth value extracted from Fig. 5. G means Gaussian beam; T means Top-hat beam.

\begin{tabular}{|c|c|c|c|c|c|c|c|}
\hline & & \multicolumn{2}{|c|}{2} & \multicolumn{2}{|c|}{4} & \multicolumn{2}{|c|}{6} \\
\hline \multicolumn{2}{|c|}{ Wavelength (nm) } & G & $\mathrm{T}$ & G & $\mathrm{T}$ & G & $\mathrm{T}$ \\
\hline \multirow[t]{2}{*}{ Penetration depth $(\mathrm{cm})$} & Fluence $=10^{-5} \mathrm{w} / \mathrm{cm}^{2}$ & 4.21 & 4.02 & 3.88 & 3.98 & 4 & 3.82 \\
\hline & Fluence $=10^{-7} \mathrm{w} / \mathrm{cm}^{2}$ & 5.68 & 5.1 & 4.88 & 4.74 & 4.92 & 4.82 \\
\hline
\end{tabular}

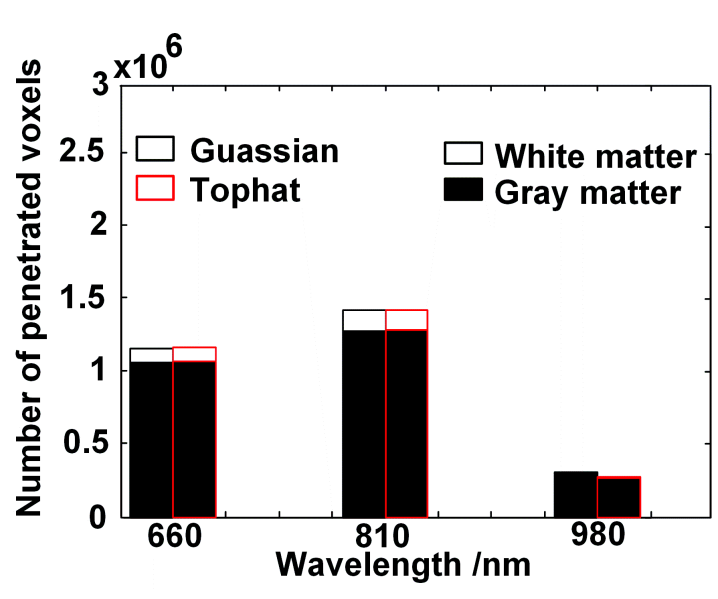

(a)

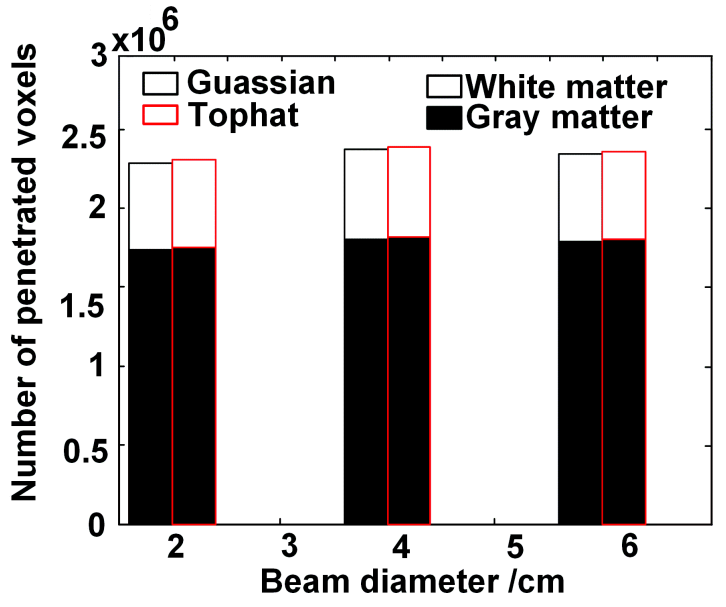

(b)

Fig. 6. (a) Mean number of light penetrated voxels within cerebral tissue respective to Gaussian and Top-hat beams at different wavelengths; (b) Mean number of penetrated voxels within brain tissue respective to Gaussian and Top-hat beams of different diameters.

for $4 \mathrm{~cm}$ and $6 \mathrm{~cm}$ sizes of beams, there is no obvious difference between the numbers of light penetrated voxels.

\section{Conclusion and Discussion}

The main goal of this research is to obtain quantitative analysis of near-infrared light penetration depth and to make comparison of light penetration depths at some commonly-used treated light properties for transcranial light stimulation and therapy. The whole research was conducted in the way of $3 \mathrm{D}$ Monte Carlo simulation with the use of the most realistic human head model, $\mathrm{VCH}$, which could provide the highest realistic human model with highest-resolution structure image dataset for us to study precise light propagation, and the most reliable 3D Monte Carlo modeling technique, which could help simulate light transportation inside human head realistically. The result we got is relatively more accurate and realistic than the previous related reports with simplistic structured or low-resolution MRI slice-based head models or light propagation computation with those method not for highly realistic head model. The light penetration depth were extracted and scaled by pie chart of accumulated fluence statistics, the light distribution of fluence in structured head profile, the sampled decline curve of fluence at light incidence direction, and voxel numbers of irradiant voxels in different types of tissues. All of the result provided successful quantitative analysis of light penetration depth for transcranial LLLT.

We explored the percentage of light fluence in different-depth tissues in the way of simulation, measured the whole number of penetrated voxels in every experimental group and compare them in histograms, etc. The numeric evaluation of light penetration depth and quantitative comparison of those beams were analyzed in two aspects: the penetration depth of single beam and the total 
number of penetrated voxels. To find the effects of factors such as wavelength, beam size and beam type, we chose different but frequently-used wavelengths $(660,810$ and $980 \mathrm{~nm})$, beam diameters $(2,4$ and $6 \mathrm{~cm}$ ) and beam types (Gaussian and Top-hat beams) and divided them into two groups, each had six simulations of two beam types and three wavelengths or beam sizes.

In this study, we made quantitative investigation on stimulated/treated light penetration depth, which were mostly qualitatively studied by previous report and accordingly rarely reliable quantitative evaluation were reported. Our study showed that $810 \mathrm{~nm}$ was shown to be the most ideal choice for transcranial LLLT treatment for the widest and deepest penetration. $980 \mathrm{~nm}$ is shown to be suitable for light acupuncture since the light at this wavelength is more concentrated and penetrates lightly. As for beam diameter, $2 \mathrm{~cm}$ size of light also performs better than those of $4 \mathrm{~cm}$ and $6 \mathrm{~cm}$ in penetrating within brain, but the difference between them is not quite significant. The similar finding was also reported by others before, ${ }^{15}$ however, in this research we went further by quantitative study. We observed the exact penetration depth of each beam and the total number of penetrated voxels. For example, the number of voxels penetrated by $660-\mathrm{nm}$-light is about $4 / 5$ of $810 \mathrm{~nm}$, and that of $980-\mathrm{nm}$-light is about $1 / 5$ of that of 880 -nm-light. Taken together of all quantitative analysis of all results in this study, it is distinct that wavelength takes the largest effect on penetration depth, the second is beam size and the third is beam type.

Limited by undoable experimental test of light penetration depth for clinical transcranial LLLT, we were not allowed to verify our simulation results but compare it with some other researchers' report. However, the simulation method, we used is of high accuracy and has been fully tested. MCVM is capable of precise modeling light propagation in 3D structured media; and the $\mathrm{VCH}$ is the top realistic human model with highest resolution and finest segmentation referring to tissue type. In the future, we may perform relevant experiments on animals to measure photon penetration depth for light stimulation and therapy for brain diseases and address to current issues on light distribution ${ }^{21} /$ diseases $^{22}$ with our MCVM-VCH method.

Taken together, by using Monte Carlo Modeling method for $3 \mathrm{D}$ voxelized media and $\mathrm{VCH}$ head dataset, we obtained reliable light fluence distribution at different illumination parameters combinations. We surprisingly found that $810 \mathrm{~nm}$ light enabled maximum penetration depth $(>5 \mathrm{~cm})$ penetration depth when beam size is smaller than $2 \mathrm{~cm}$, no matter what type of beam profile. It means that light stimulation at such illumination set allowed light crossing through gray matter into white matter, which supports the therapeutic effect of low-level therapy for brain function deficit or dysfunction. Additionally, the quantitative evaluation of light penetration depth at commonly used illumination parameter range is very helpful for researchers and clinicians guiding and optimizing the light stimulation and parameters, precision or personal medicine, and safety control. This study advantageously offered us reliable and quantitative evaluation of photon penetration depth for light stimulation and treatment field of biomedical optics. Plus, it showed us an innovative, fast, low cost, nonanimal-test way to achieve light penetration depth at actually used illumination properties for research and clinics.

\section{Acknowledgment}

We thank the supports of the One University One Zone Growth Fund (No. A03013023001019), the Central University Basic Scientific Research Project Business Expenses (No. ZYGX2016J052), CAMS Innovation Fund for Medical Sciences (No. 2016-I2M-3-023) and National Natural Science Fund Projects (No. 61675039).

\section{References}

1. Q. Wu, Y. Huang, S. Dhital, S. K. Sharma, A. C. Chen, M. L. Whalen, M. R. Hamblin, R. W. Waynant, J. Anders, "Low level laser therapy for traumatic brain injury," Proc. of SPIE 755206-8, 151-154 (2010).

2. K. M. Ashok, A. Kumar, N. A. Moussa, "Low-level laser therapy: A useful technique for enhancing the proliferation of various cultured cells," Lasers Med. Sci. 27, 237-249 (2012).

3. G. Arias-Gil, F. W. Ohl, K. Takagaki, M. T. Lippert, "Measurement, modeling, and prediction of temperature rise due to optogenetic brain stimulation," Neurophotonics 3, 045007 (2016).

4. T. Li, C. Cao, H. Gong, "Study on the detection depth of NIRS system in biological tissue," $J$. Biomed. Eng. Res. 25, 32-35 (2006). 
5. H. Jia, B. Chen, D. Li, Y. Zhang, "Boundary discretization in the numerical simulation of light propagation in skin tissue: Problem and strategy," J. Biomed. Opt. 20, 25007 (2015).

6. L. Wang, S. T. Jacques, L. Q. Zheng, "MCML Monte Carlo modeling of light transport in multilayered tissues," Comp. Methods Program. Biomed. 47, 131-146 (1995).

7. D. Boas, J. Culver, J. Stott, A. Dunn, "Three dimensional Monte Carlo code for photon migration through complex heterogeneous media including the adult human head," Opt. Express 10, 159-170 (2002).

8. L. H. V. Wang, R. E. Nordquist, W. R. Chen, "Optimal beam size for light delivery to absorptionenhanced tumors buried in biological tissues and effect of multiple-beam delivery: A Monte Carlo study," Appl. Opt. 36, 8286-8291 (1997).

9. H. Gong, Q. Luo, T. Li, "MCVM: Monte Carlo modeling of photon migration in voxelized media," J. Innov. Opt. Health Sci. 3, 91-102 (2010).

10. T. Li, H. Gong, Q. Luo, "Visualization of light propagation in visible Chinese human head for functional near-infrared spectroscopy," J. Biomed. Opt. 16, 045001 (2011).

11. T. Li, Y. Li, Y. Sun, M. Duan, L. Peng. "Effects of head models on Monte Carlo simulation of light propagation for functional near-infrared spectroscopy," J. Innova. Opt. Health Sci. 8, 1550024 (2015).

12. P. J. Muller, B. C. Wilson, "An update on the penetration depth of $630 \mathrm{~nm}$ light in normal and malignant human brain tissue in vivo," Phys. Med. Biol. 31, 1295-1297 (1986).

13. L. Yue, M. S. Humayun, "Monte Carlo analysis of the enhanced transcranial penetration using distributed near-infrared emitter array," J. Biomed. Opt. 20, 88001 (2015).

14. C. Thunshelle, M. R. Hamblin, "Transcranial lowlevel laser (Light) Therapy for Brain Injury," Photomed. Laser Surg. 34, 587-598 (2016).
15. Y. Zhao, T. Li, Y. Sun, K. Li, "Effects of wavelength, beam type and size on cerebral low-level laser therapy by a Monte Carlo study on visible Chinese human," J. Innov. Opt. Health Sci. 8, 1540002 (2014).

16. J.-X. Dai, M. S. Chung, R. M. Qu, L. Yuan, S. W. Liu, D. S. Shin, "The visible human projects in Korea and China with improved images and diverse applications," Surg. Radiol. Anat. 34, 527-534 (2012).

17. S. X. Zhang, P. A. Heng, Z. J. Liu, L. W. Tan, M. G. Qiu, Q. Y. Li, R. X. Liao, K. Li, G. Y. Cui, Y. L. Guo, X. P. Yang, G. J. Liu, J. L. Shan, J. J. Liu, W. G. Zhang, X. H. Chen, J. H. Chen, J. Wang, W. Chen, M. Lu, J. You, X. L. Pang, H. Xiao, Y. M. Xie, J. C. Cheng, "The Chinese Visible Human $(\mathrm{CVH})$ datasets incorporate technical and imaging advances on earlier digital humans," J. Anatomy 204, 165-173 (2004).

18. G. Zhang, Q. Liu, Q. Luo, "Monte Carlo simulations for external neutron dosimetry based on the visible chinese human phantom," Phys. Med. Biol. 52, 7367-7383 (2007).

19. A. N. Yaroslavsky, P. C. Schulze, I. V. Yaroslavsky, R. Schober, F. Ulrich, H. J. Schwarzmaier, "Optical properties of selected native and coagulated human brain tissues in vitro in the visible and near infrared spectral range," Phys. Med. Biol. 47, 2059-2073 (2002).

20. C. R. Simpson, "Near-infrared optical properties of ex vivo human skin and subcutaneous tissues measured using the Monte carlo inversion technique," Phys. Med. Biol. 43, 2465-2478 (1998).

21. K. Zhou, J. Tian, Q. Zhang, X. Meng, K. Yang, Q. Ren, "Simulation and quantitative analysis of fluorescence intensity distribution based on the Monte Carlo method," J. Innov. Opt. Health Sci. 8, 1550038 (2015).

22. Y. Liu, H. Wang, Y. Liu, W. Li, Z. Qian, "Monte Carlo and phantom study in the brain edema models," J. Innov. Opt. Health Sci. 10, 1650050 (2017). 